\title{
INFLUÊNCIA DA TEMPERATURA NA REOLOGIA DE FLUIDOS DE PERFURAÇÃO PREPARADOS COM CARBOXIMETILCELULOSE, GOMA XANTANA E BENTONITA
}

\author{
K. C. Melo ${ }^{1}$, T. N. C. Dantas ${ }^{2}$ e E. L. Barros Neto ${ }^{3}$ \\ ${ }^{1}$ Instituto Federal do Rio Grande do Norte \\ ${ }^{2}$ Universidade Federal do Rio Grande de Norte \\ klis.costa@ifrn.edu.br ${ }^{1}$
}

Artigo submetido em setembro/2013 e aceito em outubro/2013

\section{RESUMO}

O comportamento reológico dos fluidos de perfuração pode sofrer alterações ao longo da perfuração em função do gradiente de temperatura do poço. A degradação térmica sofrida por alguns aditivos utilizados como viscosificantes restringe a utilização da classe dependendo da temperatura a qual será exposto. Este trabalho apresenta um estudo sobre o comportamento reológico da Goma Xantana (GX), da Carboximetilcelulose (CMC) e da Bentonita (BNT) em função da temperatura. Os Modelos matemáticos de Ostwald de Waale e o de Herschel-Bulkley foram aplicados para determinar os parâmetros reológicos. 0 efeito da hidratação dos polímeros e da argila na reologia de soluções aquosas foram previamente estudados. A caracterização reológica foi realizada através de ensaios de reologia, utilizando sistema de cilindros coaxiais, para obtenção das curvas de fluxo. A reologia foi avaliada em função da temperatura para cada um dos aditivos utilizados. Os resultados mostraram que a CMC se solubiliza rapidamente em água, porém apresenta maior degradação quando exposta a temperatura mais elevada. A GX se mostrou apta para ser utilizada em formulações de fluidos que serão usados em poços com gradiente de temperatura mais elevados. A bentonita não apresentou alterações no comportamento reológico em função do gradiente de temperatura estudado.

PALAVRAS-CHAVE: Fluidos de Perfuração, Reologia, Temperatura, viscosificantes (Carboximetilcelulose, Goma Xantana e Bentonita)

\section{INFLUENCE OF TEMPERATURE ON THE RHEOLOGY OF DRILLING FLUIDS PREPARARED WITH CARBOXYMETHYLCELLULOSE, XANTANA GUM AND BENTONITE}

\begin{abstract}
The rheological behavior of drilling fluids may be altered during the perforation due to the temperature gradient of the wellbore. Thermal degradation suffered by certain additives used as viscosifiers restricts the use of the group depending on the change in temperature which it will be exposed to. This paper presents a study about the rheological behavior of xanthan gum polymer, carboxymethylcellulose (CMC), and bentonite as a function of temperature. Mathematical models like Ostwald de Waale and Herschel-Bulkley were applied to determine parameters of the rheological behavior. The hydration effect of polymers and clay in the rheology of aqueous solution was previously studied. The
\end{abstract}

rheological characterization was assessed through rheological trials using a system of coaxial cylinders to obtain flow curves. The rheology was evaluated as a function of temperature for each additive used. The results showed that carboxymethylcellulose quickly solubilizes in water but exhibits a greater degradation when exposed to higher temperatures. The xanthan gum polymer proved to be suitable for the formulation of fluids which will be used in wellbores with a higher temperature gradient. Additionally, bentonite did not present any change in the rheological behavior as a function of the temperature gradient studied.

KEYWORDS: Drilling Fluids, Rheology, Temperature, viscosifiers (Carboxymethylcellulose, Xanthan Gum and Bentonite). 


\section{INFLUÊNCIA DA TEMPERATURA NA REOLOGIA DE FLUIDOS DE PERFURAÇÃO PREPARADOS COM CMC, GX E BENTONITA}

\section{INTRODUÇÃO}

O fluido de perfuração, utilizado na construção de poços de petróleo e comumente conhecidos como "lama de perfuração", é o único componente que permanece em contato com o reservatório durante toda a operação de estruturação do poço. O sistema fluido é desenvolvido para atuar de forma eficiente em função das características esperadas para cada intervalo de formação. Esse sistema compreende um volume de fluido, que é bombeado da superfície até a broca pelo interior da coluna de perfuração, retornando pelo anular para a superfície onde atravessa os equipamentos retentores de sólidos e passa por tratamentos indispensáveis à sua manutenção, quando necessário (LAKE, 2006).

Os fluidos podem ser classificados segundo o constituinte principal da fase contínua (ou dispersante) em fluidos de base aquosa (ou base água) e fluidos de base não aquosa (ou base óleo). Eles são tradicionalmente classificados como fluidos base gás, fluidos base óleo, fluidos base água, sendo os dois últimos os mais utilizados (DARLEY e GRAY, 1988; FÉLIX et al., 2007; THOMAS, 2001).

De acordo com (DARLEY e GRAY, 1988) nos fluidos de base aquosa tem-se partículas sólidas suspensas em água, e o óleo pode ser emulsionado na água, nos casos em que a água é denominada como a fase contínua. Nos fluidos de base não aquosa, os sólidos são suspensos em óleo, e a água pode ser emulsionada no óleo, no caso do óleo ser a fase contínua. Nos fluidos de base gás, os cortes perfurados são removidos pelo fluxo de ar ou gás natural em alta velocidade. Agentes espumantes são adicionados para remover os menores influxos de água.

O comportamento de um fluido está diretamente relacionado com o desempenho de suas propriedades avaliadas na sua aplicação. Segundo (LAKE, 2006), existem algumas funções que o fluido deve desempenhar durante a perfuração, são elas: limpar o fundo do poço, transportar os cascalhos até a superfície para que possam ser separados mecanicamente, equilibrar as pressões existentes na formação minimizando o risco de kick e blow-out, estabilizar as paredes do poço, resfriar e lubrificar a coluna de perfuração e a broca, impedir ou minimizar os danos a formação, transmitir potência hidráulica à broca e permitir transmissão de informações através de dados de perfilagem.

Os fluidos exercem, devidamente, suas funções quando suas propriedades físicas e químicas são ajustadas para cada tipo de situação desejada. As propriedades físicas mais importantes e, frequentemente, medidas nas sondas são: densidade, parâmetros reológicos, forças géis (inicial e final), parâmetros de filtração e teor de sólidos. As propriedades químicas mais frequentemente determinadas nos laboratórios das sondas são: $\mathrm{pH}$, teores de cloreto, de bentonita e alcalinidade (THOMAS, 2001).

As propriedades reológicas de um fluido de perfuração descrevem as características de fluxo de um fluido, submetidos às várias circunstâncias de deformação. Em um sistema de circulação de fluido, o fluxo acontece em uma variedade de taxas nas canalizações de tamanhos e de formas diferentes. Para prever os efeitos deste fluxo é preciso saber o comportamento reológico do fluido nos vários pontos de interesse dentro do sistema de circulação (ANNIS e 
SMITH, 1996). Esse comportamento é definido pelos parâmetros reológicos, considerando que o fluido é definido como um modelo reológico, cujos parâmetros vão implicar diretamente no cálculo de perdas de cargas na tubulação e na velocidade de transporte dos cascalhos (MACHADO, 2002a).

O controle de propriedades reológicas, no fluido de perfuração, é de grande importância nas operações de perfuração, pois elas são responsáveis pela remoção dos cascalhos perfurados, minimizam a pressão nas bombas evitando o influxo e prevenindo a perda de fluido por circulação (FÉLIX et al., 2007). Além disso, influenciam no progresso da perfuração, reduzindo a taxa de penetração.

As propriedades reológicas de um fluido de perfuração são modificadas mediante exposição a altas temperaturas, de forma que suas propriedades, medidas na superfície, não serão iguais àquelas que o fluido exibe no fundo do poço, o que pode ser avaliado como uma redução de sua eficiência, tendo em vista que os fluidos são projetados com o intuito de se obter graus específicos para cada uma de suas propriedades, e qualquer alteração nestas provocará uma alteração no cenário de trabalho (MORIYAMA, 2010).

Os aditivos, frequentemente utilizados para doar viscosidade aos fluidos de perfuração, são os polímeros e argilas. A Carboximetilcelulose (CMC), a Goma Xantana (GX) e a Bentonita são viscosificantes largamente utilizados na indústria petrolífera.

A Carboximetilcelulose, normalmente encontrada na forma sódica, como carboximetilcelulose de sódio, é um polímero aniônico derivado da celulose, de baixo custo, que possui cadeia linear e é solúvel em água (NASCIMENTO et al., 2013).

A Goma Xantana é um polissacarídeo sintetizado por uma bactéria fitopatogênica do gênero Xanthomonas e tem extrema importância comercial (NASCIMENTO et al., 2013). O lugar de destaque no mercado se deve ao fato de que a GX apresenta propriedades reológicas bastante distintas e incomuns, tais como alto grau de pseudoplasticidade e elevada viscosidade, mesmo a baixas concentrações. O sucesso da goma é função de apresentar diversas vantagens como espessante, estabilizante, gelificante, agente de suspensão e floculação nas indústrias alimentícia, cosmética e petrolífera, dentre outras (ARAÙJO et al., 2005).

Bentonita é o nome genérico da argila composta predominantemente pelo argilomineral montmorilonita (55-70\%), do grupo das esmectitas, independentemente de sua origem ou ocorrência. As esmectitas possuem como características principais o alto poder de inchamento, até 20 vezes seu volume inicial, atingindo espaços interplanares de até $100 \AA$, alta área de superfície (até $800 \mathrm{~m}^{2} / \mathrm{g}$ ), capacidade de troca catiônica (CTC) na faixa de 60 a $170 \mathrm{meq} / 100 \mathrm{~g}$ e tixotropia. Estas características conferem à bentonita propriedades bastante específicas, que têm justificado uma vasta gama de aplicações nos mais diversos segmentos (SILVA, 2011).

O objetivo deste trabalho é avaliar o comportamento reológico em função do aumento da temperatura, utilizando três diferentes tipos de viscosificantes em formulações de fluidos de perfuração base água, CMC, GX e Bentonita.

\section{ASPECTOS TEÓRICOS}


Existem várias equações que podem descrever parâmetros reológicos de um fluido. Neste trabalho serão apresentados os modelos de Ostwald de Waale e de Herschel-Bulkley.

O modelo de Ostwald de Waale, também chamado de modelo de potência, requer dois parâmetros para caracterização do fluido. Entretanto o modelo de potência pode ser usado para representar fluidos pseudoplásticos $(n<1)$, um fluido Newtoniano $(n=1)$ ou um fluido dilatante ( $n>1$ ). A Equação (1) representa o modelo de potência ou Ostwald de Waale, mas só é válida para escoamento laminar (BOURGOYNE, A.T. JR.; MILLHEIM, K.K.; CHENEVERT, M.E.; YOUNG, 1986)

$$
\tau=K \dot{\gamma}^{n}
$$

Este modelo não se aplica para todo e qualquer fluido, nem a todo intervalo de taxa de cisalhamento. Entretanto, existe um número razoável de fluidos não newtonianos que apresentam comportamento de potência num largo intervalo de velocidades cisalhantes. Os parâmetros reológicos do fluido de potência são o índice de consistência $K$, que indica o grau de resistência do fluido diante do escoamento. Quanto maior o valor de K mais "consistente" é o fluido, e o índice de comportamento ou de fluxo, $n$, que indica fisicamente o afastamento do fluido do modelo newtoniano. Se o valor de " $n$ " se aproxima de 1,0 (um), o fluido está próximo do comportamento newtoniano. Um grande número de fluidos não newtonianos se comporta como pseudoplástico, isto é, $0<n>1$ (MACHADO, 2002b)

As emulsões e as soluções de polímeros ou de macromoléculas lineares são os exemplos mais típicos da indústria do petróleo de fluidos pseudoplásticos, que "afinam" quando a vazão ou a taxa de cisalhamento aumenta. O "afinamento" de muitos materiais líquidos se manifesta reversivelmente, isto é, eles recuperam a sua viscosidade original alta, quando o cisalhamento é reduzido ou cessado. Teoricamente, as partículas retornam ao seu estado natural de interação não orientada em relação às forças de cisalhamento. As gotas readquirem sua forma esférica e os agregados se reagrupam devido ao movimento Browniano (MACHADO, 2002a).

O modelo de Herschel-Bulkley é uma modificação do modelo de Ostwald de Waale em que se adicionou o parâmetro $\tau_{0}$, (limite de escoamento real). É chamado também de fluido de potência com limite de escoamento ou fluido de potência modificado e tem três parâmetros reológicos a serem levados em consideração, como mostra a Equação (2), que define a equação do modelo. Materiais típicos que exibem limite de escoamento real são dispersões de argila com polímeros, empregadas amplamente na indústria de petróleo como fluidos de perfuração (MACHADO, 2002a).

$$
\tau=K(\gamma)^{n}+\tau_{0}
$$

Machado, (2002a) afirma ainda que o modelo em questão é mais completo do que os anteriores, uma vez que a sua equação engloba três parâmetros. 


\section{MATERIAIS E MÉTODOS}

\section{Materiais}

Os aditivos empregados para formulação do sistema fluido foram: Bentonita, CMC (Carboximetilcelulose), GX (Goma Xantana), Calcita, Barita, Óxido de Magnésio, Polímero Catiônico e Cloreto de Sódio.

\section{Métodos}

\section{Soluções de viscosificantes}

Soluções dos diferentes viscosificantes, CMC, GX e Bentonita, em água foram preparadas em agitador Hamilton Beach, adicionando-se o viscosificante à água e agitando-se durante 10 minutos.

A Tabela 1 apresenta os valores percentuais dos aditivos viscosificantes (polímeros e argila) utilizados no preparo das soluções.

Tabela 1. Percentual dos viscosificantes utilizados na preparação dos fluidos

\begin{tabular}{cc}
\hline Viscosificante & Quantidade (\%) \\
\hline CMC & $0,17-0,34-0,6-0,68-0,77-0,85-1,03-1,37-1,71$ \\
GX & $0,11-0,17-0,22-0,28-0,34-0,68-0,85-1,03-1,37-1,71$ \\
Bentonita & $2,0-3,0-4,0-5,0$ \\
\hline
\end{tabular}

A análise reológica foi realizada para todas as soluções nas concentrações apresentadas na Tabela 1. Após análise dos resultados determinou-se a concentração que apresentou características reológicas adequadas para ser utilizada na formulação do fluido de perfuração e foi observado o tempo de hidratação, em intervalos de 24,48 e 72 horas, para cada um dos viscosificantes estudados.

A caracterização reológica foi feita através de análises realizadas em um reômetro de tensão controlada (Haake Mars-Controler-Thermo Scientific/ modular advanced rheometer system Cup DG-41/Messbecher DG 41), utilizando rotor cilíndrico (DG 41 DIN 53544 Titan A $25^{\circ} \mathrm{C}$ $\pm 0,1$ ) com a temperatura da placa controlada por banho (TC-502) com precisão de controle de aquecimento e resfriamento (Controlador Brookfield). A curva de fluxo gerada pelo cisalhamento das amostras foi obtida pelo método CR (taxa de cisalhamento controlada) no intervalo de 1 a $1050 \mathrm{~s}^{-1}$. Os gráficos de tensão versus taxa de cisalhamento foram gerados pelo software do equipamento, RheoWin versão 3.50.0012.

\section{Preparação dos fluidos de perfuração}

Os fluidos de perfuração foram preparados em um agitador Hamilton Beach, adicionando todos os componentes (viscosificante, adensantes, inibidores, alcalinizantes e outros) necessários a composição do fluido, agitando-o por um total de 60 minutos. Os aditivos foram incorporados 
ao fluido, em intervalos de cinco minutos de agitação para líquidos e dez minutos para sólidos. Após a agitação deixou-se em repouso por 24 horas para garantir a hidratação do polímero e/ou da argila; ao termino das 24 horas, cada sistema foi levado para agitação por 15 minutos e, posteriormente, analisado.

Os fluidos foram preparados com $0,85 \%$ de CMC, $0,68 \%$ de GX e $5 \%$ de Bentonita e em seguida os ensaios reológicos foram realizados em diferentes temperaturas $(15,25,35,45,55,65$ e $\left.75^{\circ} \mathrm{C}\right)$.

Os parâmetros reológicos, dos modelos de Ostwald de Waale e Herschel-Bulkley, foram obtidos utilizando o software do equipamento.

\section{RESULTADOS E DISCUSSÃO}

\section{Estudo reológico do Carboximetilcelulose (CMC) em função do seu percentual}

Foi realizado um estudo reológico da $\mathrm{CMC}$ em função do seu percentual no fluido de perfuração, com o objetivo de determinar o percentual mais adequado para o uso com essa finalidade. Nas Figuras 1 (A) e (B) encontram-se, respectivamente, as curvas de fluxo e as viscosidades para todos os fluidos preparados com CMC.

Os fluidos preparados com CMC apresentaram comportamento não-newtoniano, como pode ser observado na Figura 1 (A), na qual a relação de tensão cisalhante por taxa de cisalhamento não é constante. Isto ocorreu para todos os percentuais de CMC utilizados.

Ainda na Figura $1(A)$, pode-se observar que os reogramas partem da origem dos eixos, mostrando que as soluções preparadas com CMC não apresentaram resistência ao fluxo inicial, ou seja, limite de escoamento igual ou muito próximo de zero. Esse comportamento pode ser explicado em função das forças interpartículas e/ou intermoleculares fracas, formadas nas dispersões com CMC.

Observando a Figura 1 (B) percebe-se que ocorre um aumento de viscosidade com o aumento do percentual da CMC presente na solução, ou seja, o aumento da quantidade de polímero no meio promove uma estruturação das moléculas e, consequentemente, proporciona um aumento da viscosidade da solução.

Esse comportamento se justifica pelo peso molecular e o tamanho da macromolécula, que proporcionam maiores interações intermoleculares e entrelaçamentos da mesma até que a solução deixa de ser diluída e passa a ser uma solução concentrada, ponto definido como concentração crítica, que é originado pelo produto da formação de aglomerados de partículas. Nesse ponto, o movimento de uma partícula depende do movimento da adjacente (DIAZ et al., 2004). 

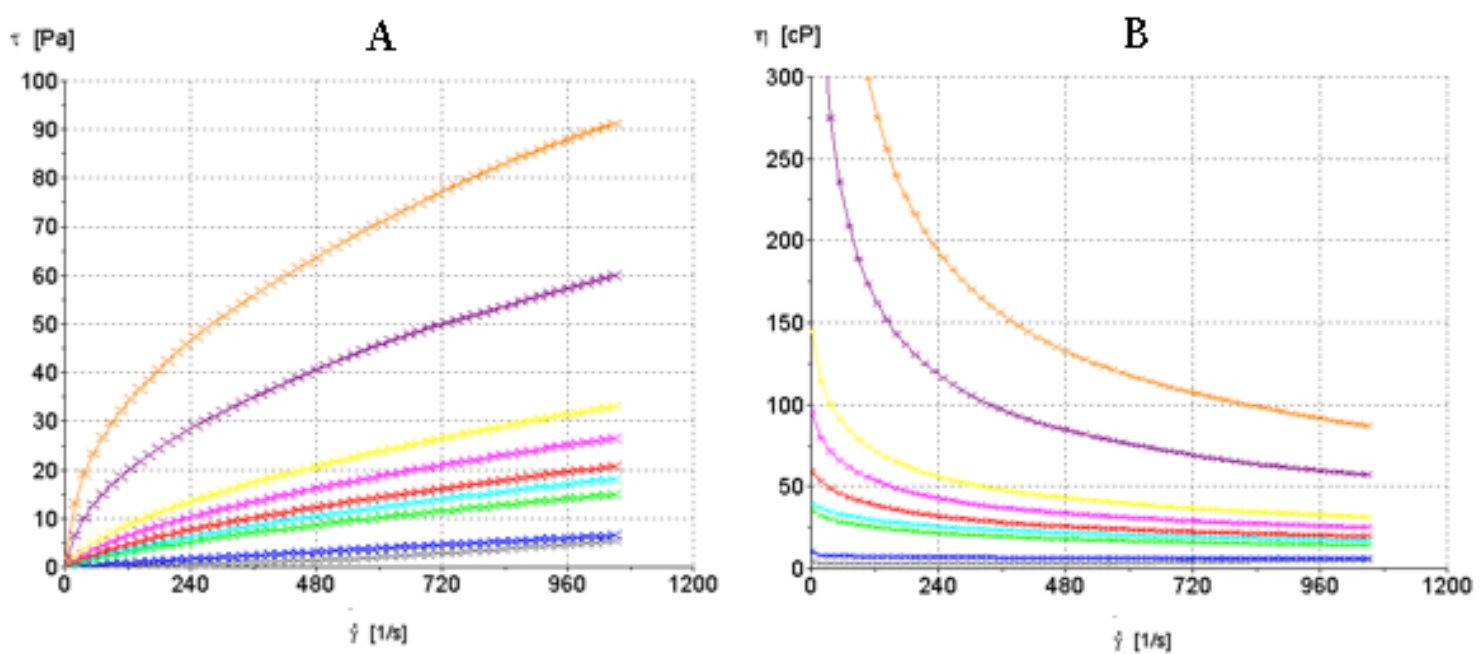

Figura 1. Curvas de fluxo (A) e viscosidade (B) em função da taxa de cisalhamento e do percentual da CMC no meio: $0,17 \%(\rightarrow), 0,34 \%(\rightarrow), 0,6 \%(\rightarrow), 0,68 \%(-), 0,77 \%(-\leftarrow), 0,85 \%(-\succ), 1,03 \%()$ ), $1,37 \%(\hookrightarrow)$ e $1,71 \%(\longrightarrow)$.

A escolha das concentrações dos viscosificantes poliméricos foi realizada tomando como base o fato de que a utilização desses viscosificantes em formulações de fluidos de perfuração não devem apresentar viscosidades muito altas ou muito baixas, ou seja, devem apresentar alta viscosidade em baixas concentrações.

Com isso, os resultados obtidos nesse estudo indicam a solução preparada com $0,85 \%$ de CMC como ideal para ser utilizada na formulação do fluido de perfuração, tendo em vista que o comportamento reológico observado para a essa solução satisfaz as condições desejadas para ser empregado na formulação do fluido de perfuração.

\section{Avaliação do tempo de hidratação (CMC)}

A hidratação do polímero corresponde ao intervalo de tempo indispensável para que este alcance a condição máxima de interação com a água. As propriedades reológicas dessas soluções irão sofrer alterações, até que se atinja esse tempo.

No intuito de avaliar o tempo necessário para que o fluido de perfuração pudesse ser analisado, foi realizado um estudo prévio de hidratação a fim de determinar o tempo ideal para que o polímero atingisse a máxima hidratação.

A Figura (2) apresenta as curvas de fluxo e viscosidade obtidas para a solução preparada com $0,85 \%$ de CMC após 72 horas com intervalos de 24 horas entre as análises. 


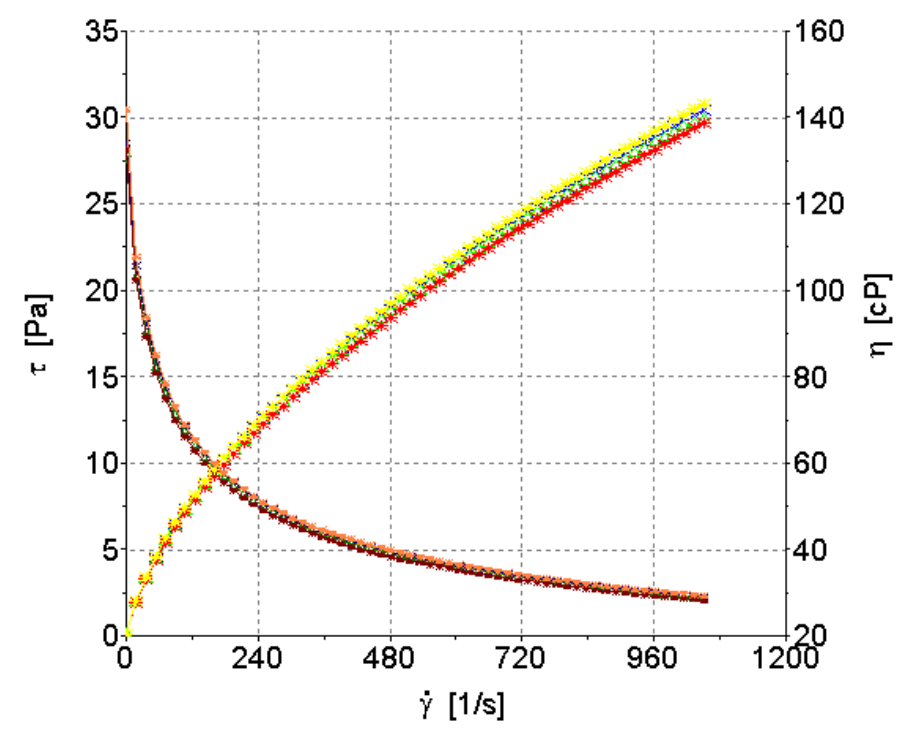

Figura 2. Curvas de fluxo e viscosidade, em função do tempo de hidratação, obtidas utilizando a solução preparada com $0,85 \%$ de CMC, em água, respectivamente:

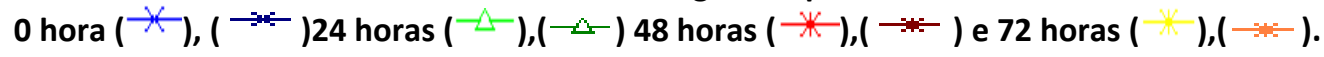

Observa-se na Figura (2) que o desempenho reológico da solução de CMC não apresentou alterações reológicas expressivas desde o seu preparo até o término das 72 horas, indicando que esse polímero tem grande potencial de solubilidade e consequente maior molhabilidade, o que permite uma hidratação rápida. Porém, é importante ressaltar que tal condição é alcançada somente mediante processo de agitação adequado.

A solubilidade da CMC pode ser explicada pela presença de sódio na molécula, que ao entrar em contato com a água se ioniza provocando a solvatação das moléculas e, consequentemente, a solubilidade no meio.

\section{Influência da temperatura na reologia do fluido preparado com CMC}

As Figuras 3(A) e (B) apresentam as curvas de fluxo e viscosidade em função da temperatura, respectivamente, para um fluido preparado com $0,85 \%$ de CMC.

Analisando a Figura 3(A), observa-se uma redução significativa nas condições reológicas do fluido de perfuração formulado com $0,85 \%$ de CMC com o aumento da temperatura. De acordo com o observado na Figura 3(B), houve uma redução na viscosidade do fluido com o aumento da temperatura.

Em soluções, a força de coesão entre as moléculas é muito maior que a transferência da quantidade de movimento (movimento Browniano) em decorrência da atividade molecular. Com isso, a tensão de cisalhamento e a viscosidade de um fluido é extremante dependente das forças de coesão que tendem a posicionar as moléculas de forma fixa e, assim, resistir ao movimento. Com o aumento da temperatura, essas forças de coesão sofrem uma redução acelerada e levam a uma redução da viscosidade (FÉLIX et al., 2007). 

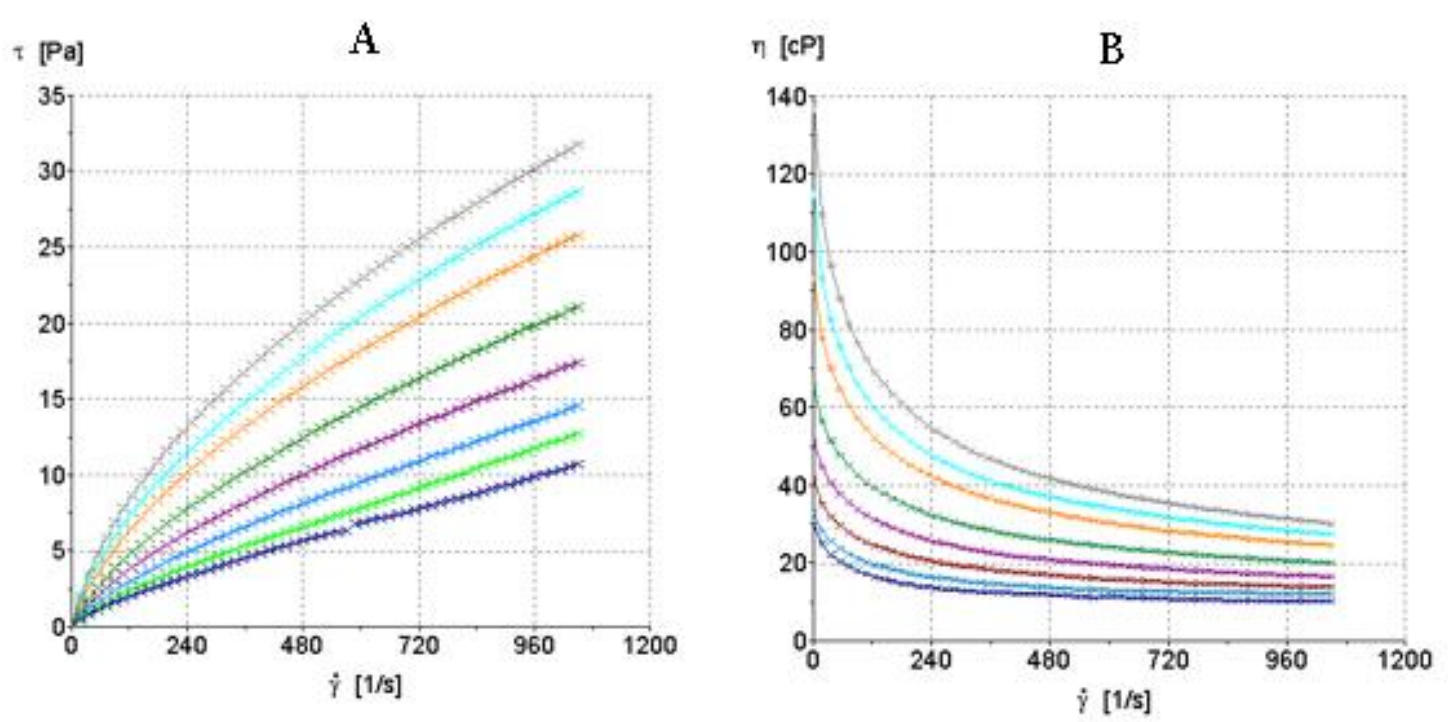

Figura 3. Curvas de fluxo (A) e viscosidade (B) em função da temperatura de operação para um fluido preparado com $0,85 \%$ de CMC, respectivamente: $15^{\circ} \mathrm{C}(\rightarrow)(-<), 25^{\circ} \mathrm{C}(\rightarrow)(-<), 35^{\circ} \mathrm{C}(\rightarrow \leftarrow)(-<), 45^{\circ} \mathrm{C}(\rightarrow \leftarrow)(\rightarrow)$, $55^{\circ} \mathrm{C}(\rightarrow)(-), 65^{\circ} \mathrm{C}(\rightarrow)(-\leftarrow)$,
$75^{\circ} \mathrm{C}(\rightarrow)(-), 85^{\circ} \mathrm{C}(\rightarrow)(\rightarrow)$

As curvas de fluxo da Figura 3(A) foram ajustadas pelo modelo de Ostwald de Waale. Os parâmetros desse modelo foram obtidos para o fluido preparado com $0,85 \%$ de CMC e estão apresentados na Tabela 2.

Tabela 2. Valores de coeficiente linear e parâmetros do modelo de Ostwald de Waale para um fluido preparado com $0,85 \%$ de CMC em função da temperatura.

\begin{tabular}{cccc}
\hline \multicolumn{4}{c}{ Modelo de Ostwald de Waale } \\
$\mathrm{T} /{ }^{0} \mathrm{C}$ & $k$ & $\mathrm{n}$ & $\mathrm{R}$ \\
15 & 0,4694 & 0,6068 & 0,9997 \\
25 & 0,3008 & 0,6408 & 0,9998 \\
35 & 0,1835 & 0,6824 & 0,9998 \\
45 & 0,1296 & 0,7042 & 0,9999 \\
55 & 0,0874 & 0,7341 & 0,9999 \\
65 & 0,0489 & 0,7965 & 0,9994 \\
75 & 0,0444 & 0,7858 & 0,9997 \\
\hline
\end{tabular}

Este modelo apresentou coeficientes de correlação $(R)$, muito próximo da unidade para todas as temperaturas analisadas, como apresentado na Tabela 2, mostrando que o mesmo se adequou bem aos dados obtidos experimentalmente. $O$ aumento na temperatura fez com que 0 fluido tendesse para o comportamento Newtoniano $(n=1)$, apresentando valores de " $n$ " crescentes. $O$ índice de consistência $k$ diminui com o aumento da temperatura, como o valor deste índice está diretamente ligado com a "consistência" do fluido, pode-se observar, na Figura $3(B)$, que a viscosidade diminui com o aumento da temperatura (Tabela 2 ).

Sugere-se que à medida que a temperatura aumenta, as interações das moléculas de CMC com a água aumentam, desestruturando o fluido como um meio homogêneo passando assim a um comportamento tendendo ao Newtoniano com o acréscimo da temperatura. 


\section{Estudo reológico da Goma Xantana (GX) em função da sua concentração}

Foi realizado um estudo reológico da GX em função do seu percentual na solução com o objetivo de determinar o percentual mais adequado para o uso na formulação do fluido de perfuração. Nas Figuras 4(A) e (B) encontram-se, respectivamente, as curvas de fluxo e as de viscosidades para os fluidos preparados com GX.
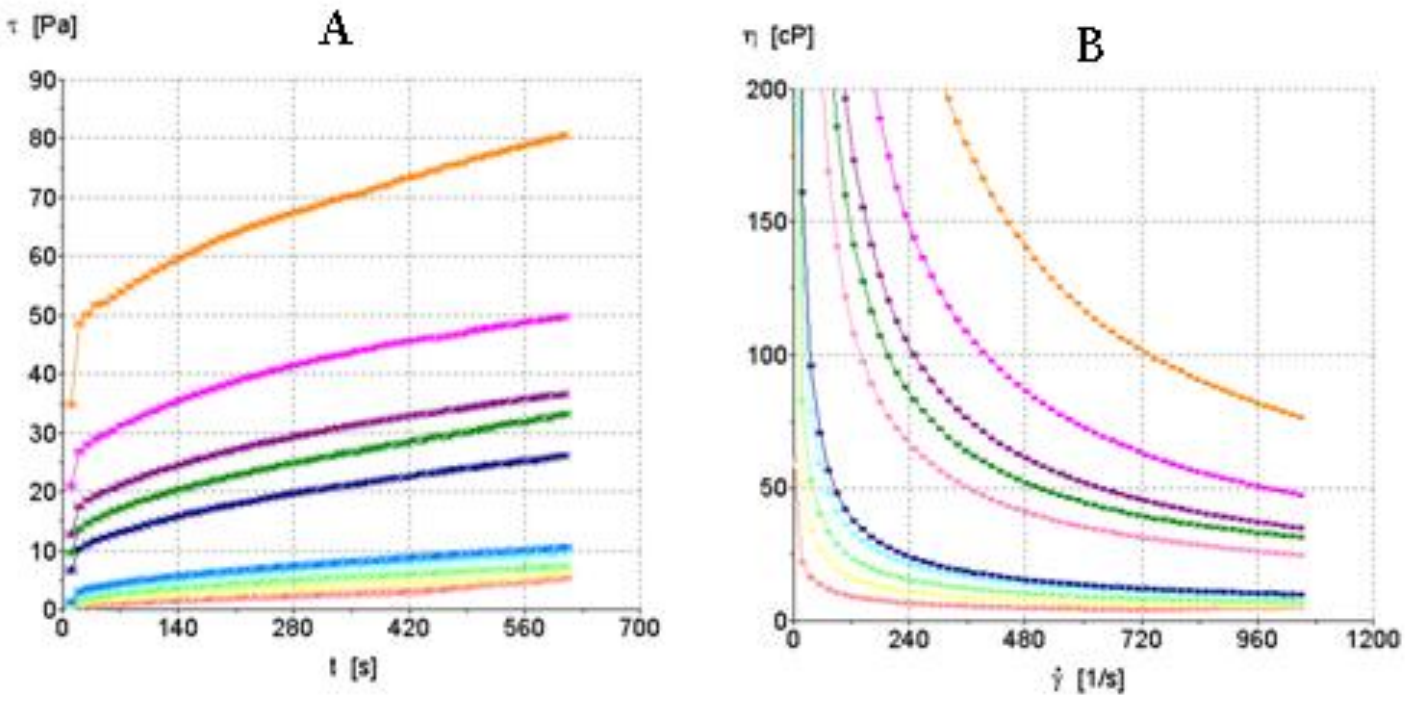

Figura 4. Curvas de fluxo (A) e viscosidade (B) em função da taxa de cisalhamento e do percentual de GX no meio:
$0,11 \%(\div), 0,17 \%($
)$, 0,22 \%(-0,28 \%($
),0,34\%(
), $0,68 \%(*-), 0,85 \%(*-)$,
$1,03 \%(*), 1,37 \%(-*), 1,71 \%(-*)$ ).

Os fluidos preparados com GX apresentaram comportamento não Newtoniano como pode ser observado na Figura 4(A), comportamento semelhante ao encontrado com as soluções de CMC. Observa-se, também, que o aumento do percentual de GX no meio oferece ao fluido um acréscimo no limite escoamento inicial.

Observa-se na Figura 4(B), que a viscosidade do fluido aumenta com o acréscimo do percentual de GX no meio e diminui com o aumento da taxa de cisalhamento, o que caracteriza este polímero como agente modificador reológico com características pseudoplásticas. Em contrapartida, ao observado para os fluidos preparados com CMC em relação ao limite de escoamento, a GX apresentou este parâmetro com bastante evidência, aumentando com o aumento do percentual deste polímero no meio. Isto mostra que as interações intramoleculares da GX são bem mais fortes que as do CMC.

É importante ressaltar, ainda, que o potencial viscosificante da GX é maior que a da CMC quando utilizadas em mesma concentração. De acordo com DIAZ et al., (2004), algumas pesquisam relatam que a viscosidade de um polímero ramificado, como é o caso da GX, é maior que a obtida para polímeros lineares nas mesmas concentrações, devido ao aumento das ramificações aumentarem quando as ramificações são longas e enrolam-se entre as cadeias. 


\section{Avaliação do tempo de hidratação (GX)}

A Figura 5 apresenta as curvas de fluxo e viscosidade obtidas para a solução preparada com $0,68 \%$ de GX após 72 horas, com intervalos de 24 horas entre as análises.

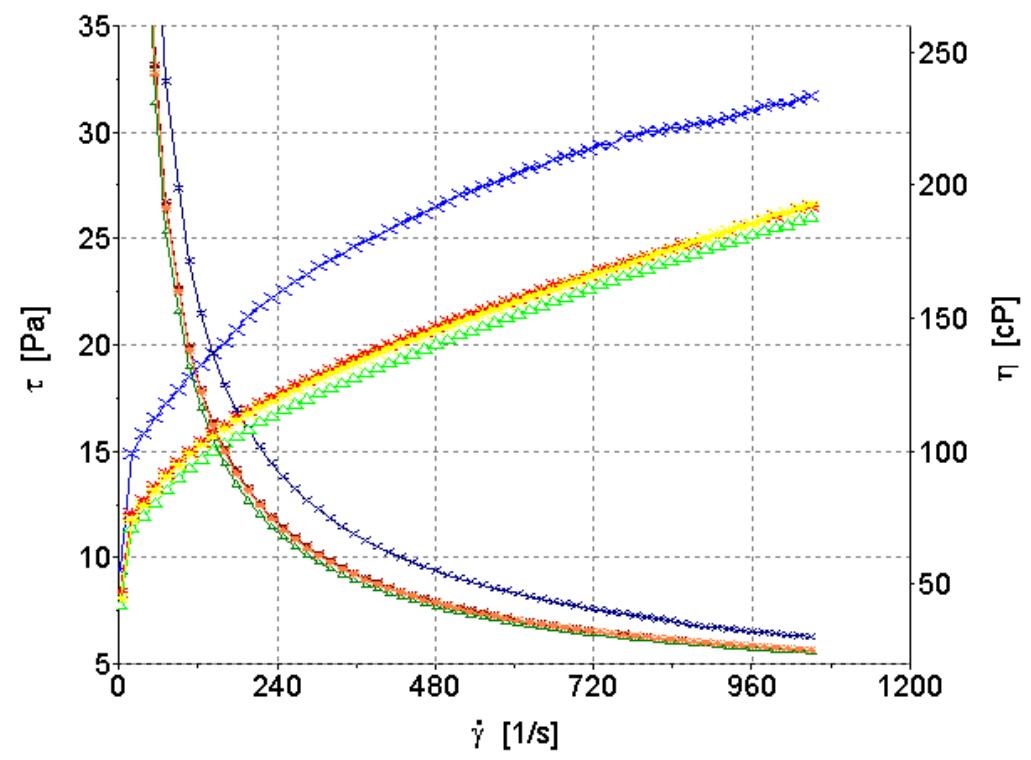

Figura 5. Curvas de fluxo e viscosidade, em função do tempo de hidratação, obtidas utilizando a solução preparada com $0,68 \%$ de GX, em água, respectivamente: 0 hora ( $\rightarrow$ ), ( $\rightarrow$ )

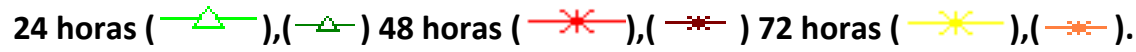

A solução preparada com $0,68 \%$ de GX exibiu uma diminuição aparente na viscosidade do fluido após 24 horas de seu preparo, como pode ser observado na Figura (5). Durante o experimento, ocorreu a incorporação de bolhas de ar ao fluido com a agitação, que desapareceu depois das primeiras 24 horas. A alta viscosidade adquirida pelo fluido com a adição da GX, aliada à alta velocidade de agitação no processo de mistura interfere na observação do efeito do tempo de hidratação nas primeiras horas. Para evitar este problema, é aconselhável a adição de um agente antiespumante ao fluido de perfuração.

O processo de hidratação polimérica é atribuído à completa dispersão do polímero no meio aquoso. Isso ocorre quando as cadeias poliméricas são solvatadas pelas moléculas de água devido ao rompimento das pontes de hidrogênio. Segundo DIAZ et al., (2004) as cadeias trissacarídicas laterais presentes na GX conferem solubilidade em meio aquoso.

Com os resultados obtidos no estudo do comportamento reológico, em função do percentual de GX presente no fluido de perfuração, escolheu-se o fluido com percentual de $0,68 \%$ de GX em função do comportamento reológico obtidos por essa solução e a viscosidade apresentada, para verificar a influência da temperatura em sua reologia.

\section{Influência da temperatura na reologia do fluido preparado com GX}

Nas Figuras 6(A) e (B) encontram-se, respectivamente, as curvas de fluxo e as viscosidades para os fluidos preparados com $0,68 \%$ de $\mathrm{GX}$. 

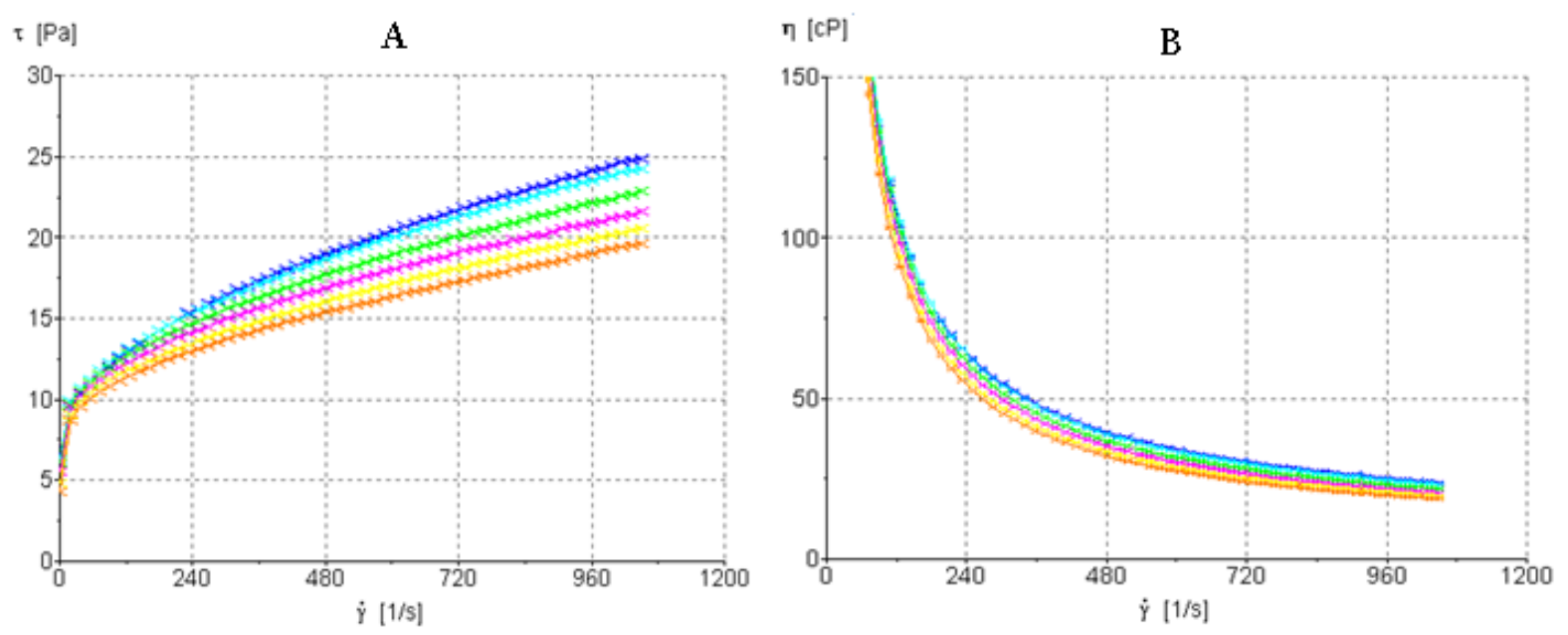

Figura 6. Curvas de fluxo (A) e viscosidade (B) de um fluido preparado com $0,68 \%$ de $\mathrm{GX}$ em função da

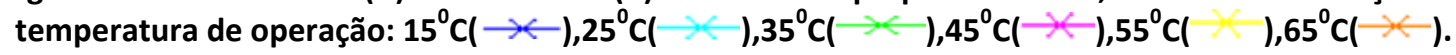

Analisando a Figura 6(B) e a Tabela 3, que apresenta os parâmetros do modelo de Herschell-Bulkley para o fluido preparado com $0,68 \%$ de $G X$, pode-se concluir que o limite de escoamento $\left(\tau_{0}\right)$ do fluido diminui com o aumento da temperatura, mas mantém a viscosidade, sua propriedade principal, em torno de um valor constante mesmo com o acréscimo da temperatura. $O$ índice de consistência $k$ indica que houve um pequeno decréscimo na viscosidade do fluido com o aumento da temperatura. Através do índice de comportamento, n, é possível afirmar que as características pseudoplásticas da GX são conservadas quando este é exposto a temperaturas entre $15^{\circ}$ e $65 \stackrel{\circ}{\circ}(0<n<1)$. O coeficiente de correlação $(R)$ mostra que 0 modelo representou bem as curvas obtidas experimentalmente, apresentando valores próximos da unidade.

Tabela 3. Valores de coeficiente linear e parâmetros do modelo de Herschell-Bulkley de um fluido preparado com $0,68 \%$ de GX em função da temperatura.

\begin{tabular}{ccccc}
\hline \multicolumn{5}{c}{ Modelo de Herschell-Bulkley } \\
\hline $\mathrm{T} / \stackrel{\circ}{ } \mathrm{C}$ & $\boldsymbol{\tau}_{0}$ & $K$ & $\mathrm{~N}$ & $\mathrm{R}$ \\
15 & 6,456 & 0,6339 & 0,4837 & 0,9996 \\
25 & 6,477 & 0,6957 & 0,4649 & 0,9991 \\
35 & 6,27 & 0,7448 & 0,4441 & 0,9986 \\
45 & 5,66 & 0,922 & 0,4069 & 0,9976 \\
55 & 4,991 & 1,005 & 0,3911 & 0,9966 \\
65 & 4,194 & 0,34 & 0,3538 & 0,9958 \\
\hline
\end{tabular}

\section{Estudo reológico da Bentonita em função da sua concentração}

Nas Figuras $7(A)$ e (B) encontram-se, as curvas de fluxo e de viscosidade obtidas para o fluido preparado com 2, 3, 4 e 5\% de Bentonita. 

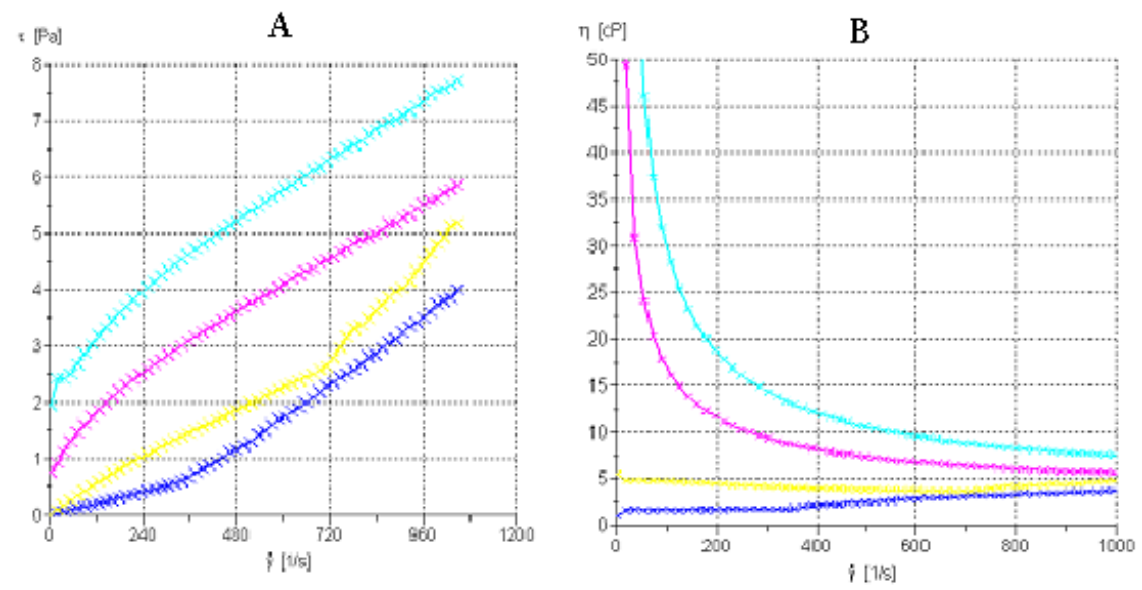

Figura 7. Curvas de fluxo (A) e viscosidade (B) obtidas para fluidos preparados com $2,0 \%(\rightarrow \leftarrow), 3,0 \%(\quad), 4,0 \%($ $\leftarrow)$ e $5,0 \%(\longleftrightarrow)$ de Bentonita.

Os fluidos aditivados com Bentonita em percentuais iguais a 2 e 3\%, apresentaram comportamento Newtoniano com mudança na inclinação da reta gerada na curva de fluxo, como pode ser observado na Figura 7(A). Tal comportamento se deve ao fato desses fluidos não apresentaram estabilidade suficiente para aumentar a viscosidade do meio, proporcionando a sedimentação das partículas sólidas presentes antes do término da análise. O comportamento Newtoniano pode ser constatado nas curvas de viscosidades, obtidas para o fluido preparado com 2 e $3 \%$ de Bentonita, Figura 7(B), que apresentaram valores constantes de viscosidade para o intervalo de taxa de cisalhamento estudado.

Os fluidos preparados com 4 e 5\%, apresentaram comportamento não-Newtoniano como pode ser observado nas Figuras 7(A) e (B), se comportando como fluidos pseudoplásticos.

A melhor condição reológica foi obtida para o fluido aditivado com $5 \%$ de Bentonita, tendo em vista que a solução apresentou maiores viscosidade e melhor estabilidade podendo ser utilizada na formulação do fluido de perfuração, para isso foi observado o tempo de hidratação dessa solução.

Avaliação do tempo de hidratação (Bentonita)

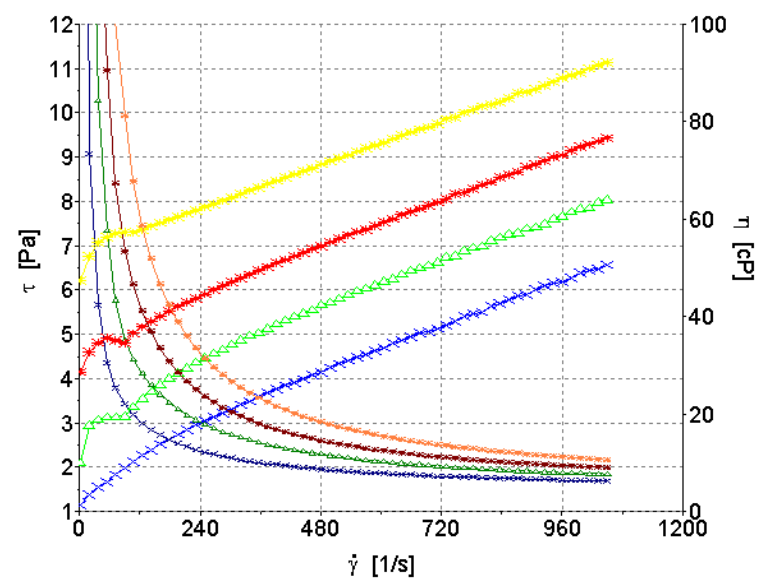

Figura 8. Curvas de fluxo e viscosidade, em função do tempo de hidratação, obtidas utilizando a solução preparada com $0,85 \%$ de Bentonita, em água, respectivamente -0 hora $(*),(\rightarrow)$

24 horas $(-\leftarrow),\left(-\triangle_{-}\right) 48$ horas $(*),(\rightarrow-) 72$ horas $(*),(-*)$. 
Na Figura (8), observa-se que a bentonita aumenta a viscosidade do meio com o tempo de hidratação. A hidratação da bentonita não foi concluída ao término das 72 horas, isso demonstra que a amostra estudada possui uma alta capacidade de inchamento. Este fato pode ser agregado a muitos fatores como natureza do cátion interplanar, carga lamelar, natureza dos sítios geradores de carga, interestratificação. Porém, o fator mais relevante apresentado na literatura costuma ser a natureza do cátion interplanar, uma vez que se o íon sódio, que se hidrata muito facilmente, estiver presente em quantidades apreciáveis, a esmectita apresenta alta capacidade de inchamento; enquanto que se outros íons, como o cálcio ou o potássio, forem predominantes, sua hidratação, pouco intensa, não permite uma grande separação das camadas (ROSSI, LUCKHAM e TADROS, 2002).

\section{Influência da temperatura na reologia do fluido preparado com Bentonita}

As Figuras 9(A) e (B) mostram, respectivamente, as curvas de fluxo e de viscosidade em função da temperatura, para uma dispersão de $5 \%$ de Bentonita em água.
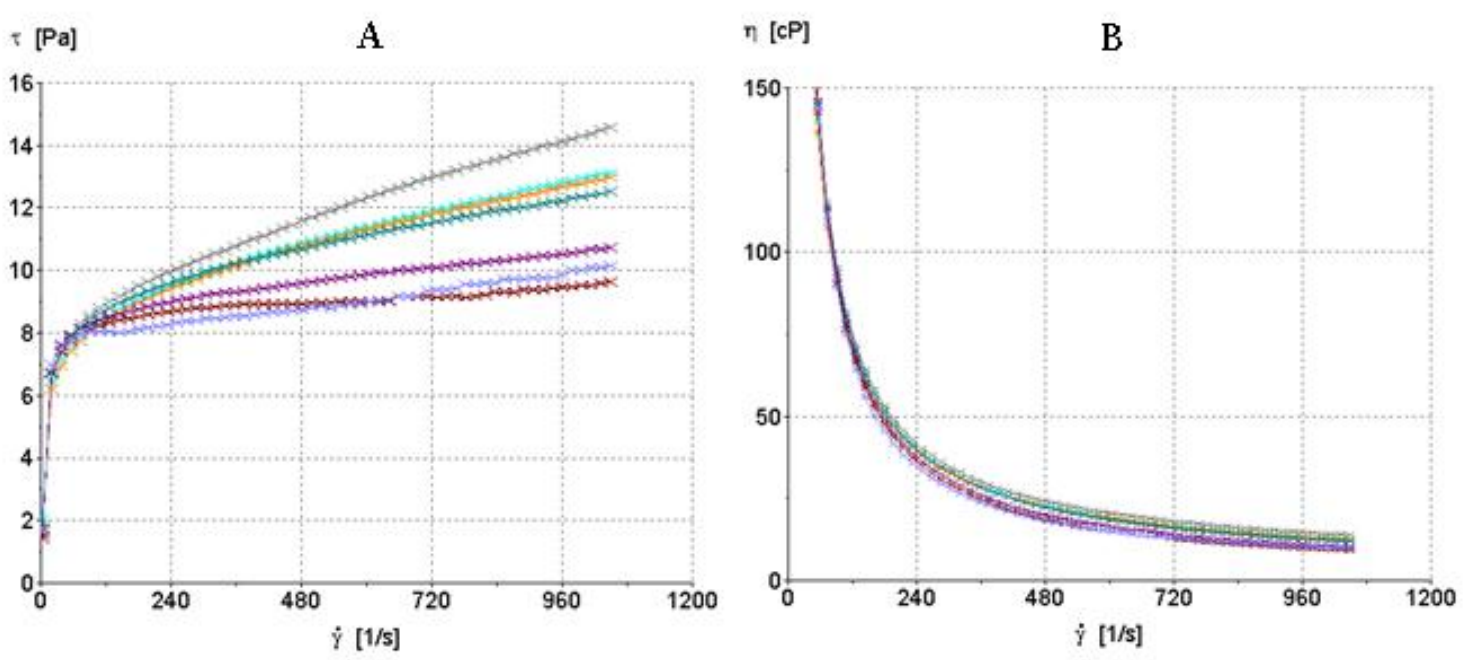

Figura 9. Curvas de fluxo (A) e viscosidade (B) de um fluido preparado com $5 \%$ de Bentonita em função da

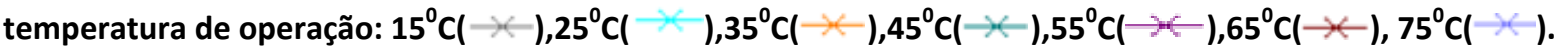

Observa-se nas curvas de fluxo geradas pelos fluidos preparados com $5 \%$ de Bentonita, Figura 9(A), que no intervalo de temperatura estudado, esse fluido apresentou alterações pouco significantes. Isso pode ser constatado analisando a Figura 9(B), que apresenta as curvas de viscosidade se sobrepondo umas às outras para as diferentes temperaturas estudadas, mostrando que apesar do aumento de temperatura não houve alterações importantes nas propriedades reológicas do fluido.

A Tabela 4 apresenta os parâmetros obtidos para o modelo de Ostwald de Waale para o fluido preparado com $5 \%$ de Bentonita.

Na Tabela 4 observa-se que o índice de consistência $k$ indica um acréscimo na viscosidade com o aumento da temperatura até $35^{\circ} \mathrm{C}$ seguido de uma redução até $45{ }^{\circ} \mathrm{C}$. O índice de fluxo indica que os fluidos apresentaram comportamento pseudoplástico, isto é, $0<n>1$. 0 coeficiente de correlação mostrou uma boa adequação dos modelos às curvas de fluxos obtidas experimentalmente. 
Tabela 4. Valores de coeficiente linear e parâmetros do modelo de Ostwald de Waale para um fluido preparado com Bentonita em função da temperatura.

\begin{tabular}{cccc}
\hline \multicolumn{4}{c}{ Modelo de Ostwald de Waale } \\
$\mathrm{T} /{ }^{\circ} \mathrm{C}$ & $k$ & $\mathrm{n}$ & $R$ \\
15 & 2,972 & 0,2241 & 0,9902 \\
25 & 3,112 & 0,2029 & 0,9928 \\
35 & 7,892 & 0,0049 & 0,8626 \\
45 & 7,683 & 0,0038 & 0,8054 \\
55 & 4,407 & 0,1274 & 0,9461 \\
65 & 4,792 & 0,1014 & 0,8834 \\
75 & 4,332 & 0,9197 & 0,9197 \\
\hline
\end{tabular}

\section{Conclusão}

O estudo da influência da temperatura no comportamento reológico dos fluidos com diferentes viscosificantes mostrou que o fluido preparado com $\mathrm{CMC}$ foi mais sensível ao aumento da temperatura que a GX e que a Bentonita não apresentou mudanças significativas em sua reologia com o aumento da temperatura.

O estudo da hidratação dos viscosificantes permitiu concluir que o CMC apresentou hidratação imediata após agitação. A GX precisou de 24 horas para estabilizar seu comportamento reológico e a Bentonita não apresentou hidratação constante ao término das 72 horas.

A GX apresentou poder viscosificante maior que o $\mathrm{CMC}$ para quantidades iguais utilizadas. Percentuais intermediários, dentre os estudados, de CMC (0,83\%) e GX (0,65\%), e percentual máximo utilizado de bentonita (5\%) apresentaram condições reológicas apropriadas para compor o fluido de perfuração.

Os modelos de Ostwald de Waale e o modelo de Herschell-Bulkley se adequaram bem aos fluidos estudados.

\section{REFERÊNCIAS BIBLIOGRÁFICAS}

1. ARAÙjO, T. A. et al. Avaliação do comportamento reológico dos diferentes meios de produção de goma xantana a partir do caldo de cana. 2005.

2. BOURGOYNE, A.T. JR.; MILLHEIM, K.K.; CHENEVERT, M.E.; YOUNG, F. S. J. Applied Drlling Engineering. International Journal of Rock Mechanics and Mining Sciences \& Geomechanics Abstracts, v. 24, n. 4, p. 149, 1986.

3. DARLEY, H. C. H. e GRAY, G. R. Composition and Properties of Drilling and Completion Fluids. Fifty ed. [S.I: s.n.], 1988. p. 654

4. DIAZ, P. S. et al. Reologia de Xantana : uma Revisão sobre a Influência de Eletrólitos na Viscosidade de Soluções Aquosas de Gomas Xantana. Semina: Ciências Exatas e Tecnológicas, v. 25, n. 1, p. 15-28, 2004.

5. FÉLIX, T. de F. et al. DESENVOLVIMENTO DE FLUIDOS DE PERFURAÇÃO À BASE DE ÁGUA COM ALTA CAPACIDADE DE INIBIÇÃO E ALTA LUBRICIDADE. $4^{\circ}$ PDPETRO, 2007. 
6. LAKE, L. W. EDPETROLEUM ENGINEERING GENERAL ENGINEERING. Richardson,TX: PETROLEUM ENGINEERING HANDBOOK, 2006. v. Ip. 864

7. MACHADO, J. C. V. Reologia e Escoamento de Fluidos - Ênfase na Indústria do Petróleo. [S.I.]: Interciência, 2002a.

8. MACHADO, J. C. V. Fluido de Perfuração. [S.I.]: Progarma Trainer Petrobras- UM$B A / S T / E P, 2002 b$.

9. MORIYAMA, A. L. L. Avaliação do Envelhecimento Dinâmico nas Propriedades de Fluidos de Perfuração Base Água. UNIVERSIDADE FEDERAL DO RIO GRANDE DO NORTE - UFRN [S.I.]. 2010.

10. NASCIMENTO, R. A. de M. et al. Degradação térmica de fluidos de perfuração argilosos aditivados com polímeros e lubrificante Thermal degradation of clay drilling fluids with polymers and lubricant additives. Matéria, v. 18, n. 2, p. 1329-1339, 2013.

11. ROSSI, S.;; LUCKHAM, P. . e TADROS, T. . Influence of non-ionic polymers on the rheological behaviour of Na+-montmorillonite clay suspensions-I Nonylphenolpolypropylene oxide-polyethylene oxide copolymers. Colloids and Surfaces A: Physicochemical and Engineering Aspects, v. 201, n. 1-3, p. 85-100, doi:10.1016/S09277757(01)00792-0, 2002.

12. SILVA, T. H. C. Da. Bentonita. Disponível em: <https://sistemas.dnpm.gov.br/publicacao/mostra_imagem.asp?IDBancoArquivoArquivo =7372>. Acesso em: 13 out. 2012.

13. THOMAS, J. E. Fundamentos de engenharia de petróleo. $2^{\circ}$. ed. Rio de Janeioro-RJ: INTERFERÊNCIA, 2001. p. 271 